\title{
Video Article \\ Laser-heating and Radiance Spectrometry for the Study of Nuclear Materials in Conditions Simulating a Nuclear Power Plant Accident
}

\author{
Dario Manara ${ }^{1}$, Luca Soldi ${ }^{1,2,4}$, Sara Mastromarino ${ }^{1,3,5}$, Kostantinos Boboridis ${ }^{1}$, Davide Robba ${ }^{1}$, Luka Vlahovic ${ }^{1}$, Rudy Konings ${ }^{1}$ \\ ${ }^{1}$ European Commission, Joint Research Centre \\ ${ }^{2}$ Energy Department, Politecnico di Milano \\ ${ }^{3}$ Department of Chemical Physics, Sapienza - Università di Roma \\ ${ }^{4}$ CEA Saclay \\ ${ }^{5}$ TU Delft
}

Correspondence to: Dario Manara at Dario.MANARA@ec.europa.eu

URL: https://www.jove.com/video/54807

DOI: doi: $10.3791 / 54807$

Keywords: Chemistry, Issue 130, High Temperature, Laser Heating, Nuclear Materials, Radiance Spectroscopy, Severe Accidents, Corium, Core Meltdown

Date Published: $12 / 14 / 2017$

Citation: Manara, D., Soldi, L., Mastromarino, S., Boboridis, K., Robba, D., Vlahovic, L., Konings, R. Laser-heating and Radiance Spectrometry for the Study of Nuclear Materials in Conditions Simulating a Nuclear Power Plant Accident. J. Vis. Exp. (130), e54807, doi:10.3791/54807 (2017).

\section{Abstract}

Major and severe accidents have occurred three times in nuclear power plants (NPPs), at Three Mile Island (USA, 1979), Chernobyl (former USSR, 1986) and Fukushima (Japan, 2011). Research on the causes, dynamics, and consequences of these mishaps has been performed in a few laboratories worldwide in the last three decades. Common goals of such research activities are: the prevention of these kinds of accidents, both in existing and potential new nuclear power plants; the minimization of their eventual consequences; and ultimately, a full understanding of the real risks connected with NPPs. At the European Commission Joint Research Centre's Institute for Transuranium Elements, a laserheating and fast radiance spectro-pyrometry facility is used for the laboratory simulation, on a small scale, of NPP core meltdown, the most common type of severe accident (SA) that can occur in a nuclear reactor as a consequence of a failure of the cooling system. This simulation tool permits fast and effective high-temperature measurements on real nuclear materials, such as plutonium and minor actinide-containing fission fuel samples. In this respect, and in its capability to produce large amount of data concerning materials under extreme conditions, the current experimental approach is certainly unique. For current and future concepts of NPP, example results are presented on the melting behavior of some different types of nuclear fuels: uranium-plutonium oxides, carbides, and nitrides. Results on the high-temperature interaction of oxide fuels with containment materials are also briefly shown.

\section{Video Link}

The video component of this article can be found at https://www.jove.com/video/54807/

\section{Introduction}

Although nuclear fission is broadly presented as a promising large-scale, practically inexhaustible energy source, its full public acceptance is still stalled by some safety, security, and safeguard risks. The experimental approach presented in this work aims at answering some fundamental materials science questions relating to one of these risks, the occurrence of severe accidents (SAs) leading to core meltdown in a nuclear power plant (NPP). This can result in a possible release of highly-radioactive material in the environment, with severe consequences, both for people's health and the country's economy. Major SAs of this type have occurred three times in NPPs, at Three Mile Island (USA, 1979), Chernobyl (former USSR, 1986), and Fukushima (Japan, 2011). Hence, NPP SAs are the focus of considerable research in a few facilities worldwide, encompassing many challenging phenomena and complicated by very high temperatures (often exceeding $3,000 \mathrm{~K}$ ) and the presence of radioactive materials.

In this scenario, a recent directive by the European Council ${ }^{1}$ requires EU countries to give the highest priority to nuclear safety at all stages of the lifecycle of a nuclear power plant. This includes carrying out safety assessments before the construction of new nuclear power plants and also ensuring significant safety enhancements for old reactors.

In this context, a controlled-atmosphere, laser-heating and fast radiance spectro-pyrometry facility ${ }^{2,3,4}$ has been implemented at the European Commission's Joint Research Centre's Institute for Transuranium Elements for the laboratory simulation, on a small scale, of NPP core meltdown. Due to the limited sample size (typically on a cm- and 0.1-g-scale) and the high efficiency and remote nature of laser heating, this approach permits fast and effective high-temperature measurements on real nuclear materials, including plutonium and minor actinide-containing fission fuel samples. In this respect, and in its capability to produce a large amount of data concerning materials under extreme conditions, the current experimental method is recognized worldwide as being unique. In fact, other complementary investigation techniques based on induction heating have been shown to suffer from the rapid high-temperature interactions between the sample material and containment ${ }^{5}$. In addition, if 
such techniques allow and mostly need larger amounts of material for analysis, they are less suited than the present method for the investigation of real nuclear materials, due to the high radioactivity and limited availability of the samples.

In the current experiments (schematized in Figure 1), a sample, mounted in a controlled-atmosphere autoclave contained in an $\alpha$-shielded glove box, is heated by a 4.5-kW Nd:YAG CW laser.

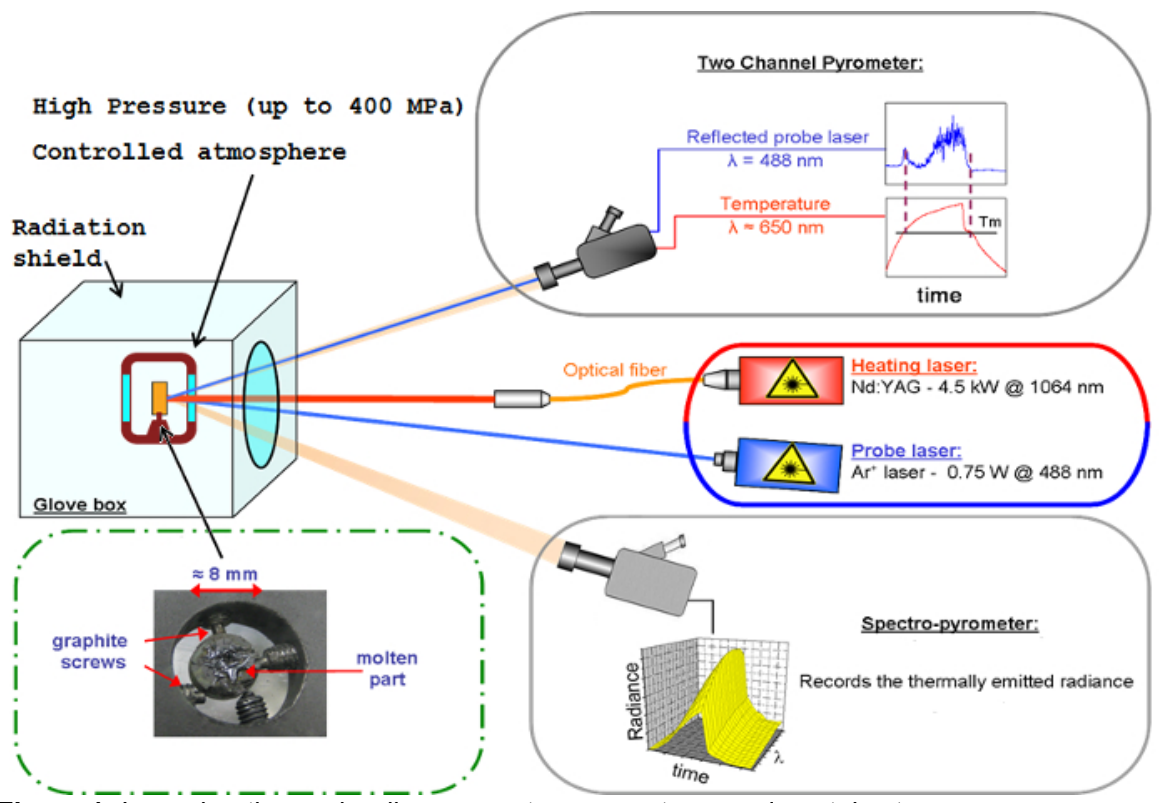

Figure $\overline{1}$ : Laser-heating and radiance spectro-pyrometry experimental set-up.

The sample is fixed with graphite (or tungsten or molybdenum) screws in a gas-tight vessel under a controlled atmosphere. The picture reported in the bottom-left corner shows, as an example, $\mathrm{a} \mathrm{PuO}_{2}$ disk fixed with graphite screws. If the sample is radioactive, the vessel should be mounted inside an alpha-tight glove box. The sample is heated by a 4.5-kW Nd:YAG laser at 1,064 nm. A fast two-channel pyrometer is used for recording the sample temperature and the reflected signal from a lower-power $\mathrm{Ar}^{+}$laser. A slower multi-channel spectro-pyromenter is employed for in situ analysis of optical properties of the hot sample. Please click here to view a larger version of this figure.

Radiation pyrometers measure the sample radiance $L_{e x}$. This is the electromagnetic radiation power density per unit surface, wavelength, and solid angle emitted by the sample at a given temperature ${ }^{6}$. It is linked to the sample surface temperature T through a modified Planck function:

$$
L_{e x}=\frac{L_{\lambda}}{c_{1}}=\frac{1}{\lambda^{5}} \cdot \frac{\varepsilon_{\lambda}(T)}{e^{c_{2}} \cdot T-1}
$$

where $L \lambda$ is the radiative power, $\varepsilon_{\lambda}$ is the spectral emissivity, $c_{1}=2 \cdot h \cdot c_{0}{ }^{2}$ is the first radiation constant, $c_{2}=h \cdot c_{0} / k_{B}=14,388 \mu \mathrm{m} \cdot \mathrm{K}$ is the second radiation constant, $c_{0}$ is the speed of light in vacuum, $h$ is Planck's constant, and $k_{B}$ Boltzmann's constant. The spectral emissivity takes into account the fact that a real body will radiate, at a given wavelength and temperature, only a fraction equal to of the power emitted by an ideal blackbody at the same temperature. Therefore, takes values between 0 and 1 , with 1 corresponding to the ideal blackbody case for which Planck's law was derived. Since pyrometers in the present work were always set up near normal with respect to the sample surface, the angle dependence of $\varepsilon_{\lambda}$ was not considered, and "emissivity" will always refer to normal spectral emissivity (NSE). The NSE must be determined in order to convert, through equation 1 and a pyrometer calibration procedure, $\mathrm{L}_{\mathrm{ex}}$ into absolute temperature $\mathrm{T}$.

The specimen temperature is detected using a fast pyrometer calibrated against standard lamps up to $2,500 \mathrm{~K}$ at $\lambda=655 \mathrm{~nm}$ and. An additional 256-channel radiance spectro-pyrometer operating between $515 \mathrm{~nm}$ and $980 \mathrm{~nm}$ was employed for the study of the NSE $\left(\varepsilon_{\lambda}\right)$ of the sample. Determination of the NSE is possible by completing a non-linear fit of the thermal emission spectrum with Equation $1^{2,3}, T$ and $\varepsilon_{\lambda}$ being the only two free parameters. This approach has been demonstrated to be acceptably accurate in refractory materials ${ }^{7}$ like those usually present in a NPP, for which the NSE can be assumed to be wavelength-independent (grey body hypothesis) on a broad spectral range. Once the temperature of the laser-heated sample is correctly measured as a function of time, thermal analysis can be performed on the resulting temperature-time curve (thermogram). Inflections or thermal arrests in the thermograms give information related to phase transitions (solidus, liquidus, and isothermal phase transformations). Moreover, besides being necessary for NSE determination, direct spectral analysis of the radiance $L_{e x}$ emitted by the hot sample also permits an in situ study of some optical properties of the studied surface. This constitutes another supporting tool for the identification of high-temperature phenomena, such as phase transitions, chemical reactions between condensed materials and the gas phase, or segregation effects. An additional technique called reflected light signal (RLS) analysis ${ }^{2,3}$ is used to confirm phase transitions. It is conducted by using the second channel of the pyrometer tuned to a low-power ( $1 \mathrm{~W}) \mathrm{Ar}^{+}$laser $(\lambda=488 \mathrm{~nm})$. This channel detects the laser beam originating from the $\mathrm{Ar}^{+}$cavity and reflected by the sample surface. A constant RLS signal indicates a solid surface, while random oscillations appear after melting due to surface tension-induced vibrations on the liquid sample surface.

In general, water-cooled reactors using solid fuel elements, currently the most common type of NPP, possess four successive barriers to ensure the containment of radioactivity ${ }^{8}$. The first barrier is that the fuel pellet itself, thanks to its crystalline structure and micro-macroscopic porosity, 
can hold the solid fission products and part of the volatile ones. In general, the entire fuel element is placed in a metallic (Zircaloy or steel) cladding that works as the second protection stage. In case of failure of the cladding, the third barrier is the whole NPP inner vessel, in general confined by a steel wall that is a few $\mathrm{cm}$ thick (primary system). Finally, the containment building (m-thick concrete) is the last safety barrier before release into the environment.

In case of failure of the water cooling system, a NPP SA can take place, leading to core overheating and meltdown. Overheating is initially due to fission heat. However, in the absence of cooling, overheating can also continue long after the termination of nuclear chain reactions, due to the residual decay heat of fission products and other highly-radioactive species contained in the nuclear core debris. In general, core melt will start from the central part of the fuel element, unless lower-melting compounds (possibly eutectics) are formed at the interface between the fuel and cladding. The first objective of the present research consists of establishing whether such lower-melting compounds can be formed in real fuelcladding systems, and, in this case, what the resulting melting temperature depression would be. In order to answer this question, the melting behavior of pure and mixed fuel compounds should first be soundly assessed, which therefore constitutes an even more important goal of the current approach. If fuel and cladding melt together, the liquid mass will rapidly fall to the bottom of the primary vessel and start reacting with the steel wall and with the remaining water and steam, if any. At this stage, steel can also be melted together with the fuel/cladding hot mixture. The resulting lava-like liquid is called "corium". This hot, highly-radioactive mixture can diffuse outside the primary containment if the steel wall is melted through and end up reacting even with the concrete constituting the most external barrier. The elevated heat and the high reactivity of the species present in the corium can lead to water dissociation and the production of hydrogen. This might result in an additional risk of steam and hydrogen explosions (cf. the SAs in Three Mile Island and Fukushima), heavy oxidation, or (less likely) hydration of the corium mass and the NPP structural materials. The current experimental method permits the separation and experimental analysis of several of the many complex physicochemical mechanisms related to the described sequence of events. Besides the mentioned pure component melting analysis and fuelcladding interaction, several high-temperature interaction mechanisms can be investigated in simplified systems, such as between Pu-containing fuel and steel, between fuel and concrete, etc. Corium formation can potentially be studied in the presence of different atmospheres (inert gas, air, traces of hydrogen or steam), producing important reference data for a comprehensive understanding of SAs.

The present approach, particularly suited for the laboratory investigation of high-melting materials, has also been employed for the successful analysis of other, more innovative types of nuclear fuels (based, for example, on uranium carbides or nitrides) and other refractory compounds, such as zirconium ${ }^{9}$, tantalum and hafnium carbides, metallic superalloys, calcium oxide ${ }^{10}$, etc.

\section{Protocol}

\section{Pyrometer and spectro-pyrometer calibration}

\section{Reference standard lamps}

1. Get certified, calibrated standard lamps from the National Standard Laboratories.

NOTE: Both lamps used here were accurately calibrated at $650 \mathrm{~nm}$ by one of the German standard reference institutions, the PTB (Physikalisch Technische Bundesanstalt, 2010).

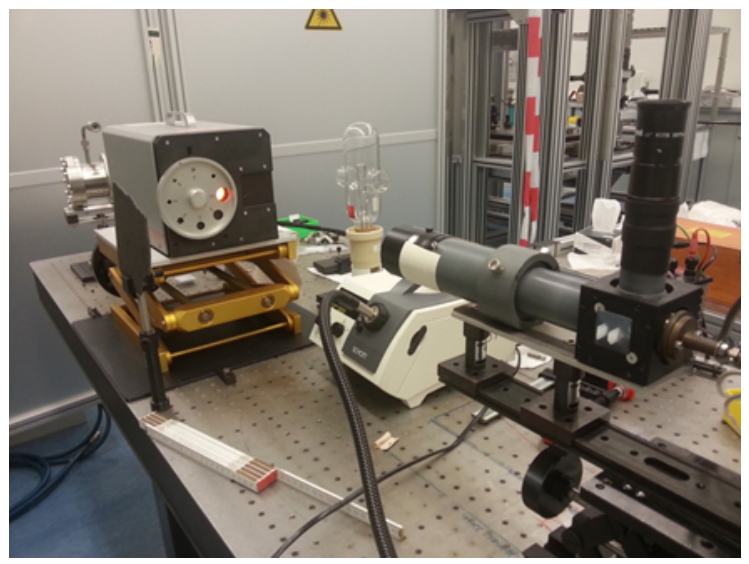

Figure 2: Blackbody source and standard lamp used for the calibration of the present pyrometer and spectro-pyrometer.

In the calibration procedure, the pyrometer or spectro-pyrometer visual field is focused on the standard light source (blackbody or lamp), which is heated to a known temperature (and therefore emits a known radiance) for a given input current. Calibration equations are obtained by fitting experimental plots of the voltage signals yielded by the pyrometer or spectro-pyrometer radiation detectors as a function of the light source temperature. Please click here to view a larger version of this figure.

\section{Pyrometer calibration}

1. Calibrate the pyrometer channel to close to $650 \mathrm{~nm}$ with two tungsten ribbon lamps, the first one for the temperature range between $1,100 \mathrm{~K}$ and $1,800 \mathrm{~K}$, and the second between $1,800 \mathrm{~K}$ and 2,500 $\mathrm{K}$ (Figure 2).

2. Align the pyrometer to the lamp filament and needle (Figure 2).

3. Record pyrometer intensities at various nominal lamp temperatures, following the calibration sheet provided by PTB.

4. Plot experimental pyrometer intensities as a function of inverse lamp temperatures.

NOTE: The pyrometer is equipped with a logarithmic amplifier. As a result, the trend should be linear, at least for temperatures above $1,700 \mathrm{~K}$. Linear fitting of experimental points will yield the pyrometer calibration equation in the form: 


$$
V_{\text {out }}(T)=A+\frac{B}{T_{\lambda}} \quad \text { Equation } 2
$$

where $A$ and $B$ are the calibration constants that permit the conversion of the pyrometer signal at $650 \mathrm{~nm}$ into the temperature.

5. Fix a second channel (photodiode) of the same pyrometer at the same wavelength of the probe laser used for the reflected light signal (RLS) analysis. In the current set-up, this is an $\mathrm{Ar}^{+} 0.75 \mathrm{~W}$ cW laser radiating at $488 \mathrm{~nm}$. Fix the pyrometer's second channel at $488 \mathrm{~nm}$ in order to allow it to function as an optical filter for the RLS.

NOTE: Since the RLS analysis is purely qualitative, no calibration is needed for this second channel.

\section{Radiance temperature}

1. Notice that $T_{\lambda}$ in Equation 2 is the radiance temperature measured by the pyrometer. It is the temperature corresponding to the experimental radiance intensity if it were emitted by an ideal blackbody $\left(\varepsilon_{\lambda}=1\right.$ in Equation 1$)$. In real samples, it is related to the real absolute temperature by the formula:

$$
\frac{1}{T}=\frac{1}{T_{\lambda}}+\frac{\lambda k_{B}}{h c_{0}} \ln \left(\varepsilon_{\lambda}\right) \quad \text { Equation } 3
$$

2. Derive Equation 3 from Equation 1 in the approximation $n \lambda T \ll h c_{0} k_{B}^{-1}$. Determine the NSE for the investigated material in order to obtain its real temperature through spectral analysis of the radiance intensity (Equation 1), recorded by a multi-channel spectropyrometer between $515 \mathrm{~nm}$ and $980 \mathrm{~nm}$.

\section{Spectro-pyrometer calibration}

1. Calibrate the spectro-pyrometer's $650-\mathrm{nm}$ channel against the channel of the standard lamp, following the same procedure explained in steps 1.2.1-1.2.3, above.

2. Since the spectro-pyrometer is not equipped with a logarithmic amplifier, but with a linear one, this time, plot the logarithm of experimental intensities (here expressed in counts) against the nominal lamp temperatures in order to obtain the calibration constants $\mathrm{C}$ and $\mathrm{D}$ for the $650 \mathrm{~nm}$ channel:

$$
\operatorname{LogI}_{\text {Connl.s }}(T)=C+\frac{D}{T_{\lambda}} \quad \text { Equation } 4
$$

3. Increase the current heating a blackbody source (Figure 2) up to a level where the blackbody cavity is luminous enough to be clearly visible with the naked eye. Then, align the spectro-pyrometer objective to the center of the blackbody cavity.

4. Increase the blackbody current to a level where the spectro-pyrometer signal, simultaneously displayed on a PC screen, is intense enough to fully cover the background noise. Adjust the spectro-pyrometer integration time in order to optimize the signal-to-noise ratio. Time-linearity of the spectro-pyrometer should be checked upon its delivery. Take care not to saturate the spectro-pyrometer photodiodes.

5. Stabilize the blackbody temperature. Wait until the blackbody emitted radiance, and therefore the spectro-pyrometer signal, is stable (typically 10 to $20 \mathrm{~min}$ at temperatures around $1,500 \mathrm{~K}$ ).

6. Record the radiance spectra on the full wavelength range spanned by the device. Fill the memory buffer entirely (256 acquisitions). Then, take average intensity values for each channel.

7. Use the intensity recorded by the channel calibrated at $650 \mathrm{~nm}$ (steps 1.4.1-1.4.2) to measure the exact blackbody temperature.

8. Once the blackbody temperature is determined, calculate the black body radiance $L_{\lambda, b b}$ using Equation 1.

9. Calibrate the remaining channels of the spectro-pyrometer against the blackbody sources (about 200 of them). Cut out the ranges between $488 \mathrm{~nm}$ and $515 \mathrm{~nm}$ and $980 \mathrm{~nm}$ and 1,011 nm to reduce noise. Obtain an integration-time-specific calibration (transfer) function

$$
K(\lambda)=\frac{L_{\lambda, b b}\left(\lambda, T_{b b}\right) \cdot t i}{\text { ICounts }_{a v}\left(\lambda, T_{b b}\right)} \quad \text { Equation } 5,
$$

where ICounts $_{a v}$ is the average experimental intensity measured by each spectro-pyrometer channel and $t i$ is the integration time. Take the average value over the 256 acquisitions accumulated in the buffer.

10. Repeat the spectro-pyrometer calibration procedure in steps 1.4.1-1.4.10 at various blackbody temperatures in order to cross check that $K(\lambda)$ is temperature-independent.

NOTE: Only the background noise should change at different blackbody temperatures.

11. In real sample measurements, obtain radiance spectra by multiplying integration-time-specific experimental radiance spectra (ICounts $(\lambda) / t i)$ by the function $K(\lambda)$.

\section{Sample mounting}

Caution: In case the sample is radioactive, carry out the entire procedure in an alpha-tight glove box equipped with optical-quality windows and an optical table. In case the sample is particularly radioactive (containing strong $\mathrm{y}$-emitters such as Pu or Am), wear a lead gown and lead gloves while mounting it. Use a radiation detector to identify the main directions of $\beta$ and $y$ irradiation.

1. Mount the sample in the holder by fixing it with graphite, molybdenum, or tungsten screws (see the bottom left inset of Figure 1). 
NOTE: Compared to graphite, Mo or W screws ensure better mechanical stability, although they can affect the sample's thermal balance more than graphite screws. The ideal sample shape is a disk approximately $8 \mathrm{~mm}$ in diameter and at least $2 \mathrm{~mm}$ thick. However, the use of adjustable screws permits the analysis of samples of different shapes and sizes that also very small and irregular. This flexibility is particularly advantageous whenever radioactive fragments are to be investigated.

2. Place the sample and the holder in a cylindrical pressure vessel (or autoclave, as schematized in Figure 1). Mount the sample perpendicularly to the vessel axis. Enclose the vessel with optical-quality windows (typically quartz or fused silica).

3. Fix the vessel to an optical table.

4. Fix a graphite screen by the rear side of the pressure vessel on an optical table in order to absorb the laser beam in case the sample should fall from the holder during an experiment.

\section{Laser and pyrometer alignment}

\section{Laser alignment}

1. On an optical table, couple a focusing unit with the fiber optics that convey the high-power laser beam to the laboratory. NOTE: In doing so, pay maximum attention to avoid the formation of kinks in the fiber optics, which may result in irreversible damage to them.

2. Choose the proper lenses in the focusing unit in order to obtain the required laser spot size on the sample surface and a suitable focal distance between the focusing unit and the sample surface. Make sure that the laser spot size is at least ten times larger than the pyrometer sighting spot (around $3 \mathrm{~mm}^{2}$ ) in order to ensure temperature homogeneity around the pyrometer measurement point. NOTE: With this restriction, the laser spot size can be adjusted according to the aims of every specific experiment. For example, a smaller spot will lead to a higher laser power density. Therefore, it will be possible to reach higher temperatures, but on a more limited zone of the sample surface. Conversely, a larger laser spot will guarantee a more homogeneous temperature distribution throughout the sample, although lower maximum temperatures will be attainable. The focal distance between the focusing unit and the sample surface is only imposed by geometrical constrictions, such as the disposition of the various optical components, the presence of a glove box wall between them and the sample, etc.

3. Mount all the optical parts needed (laser optics, $\mathrm{Ar}^{+}$laser for the RLS analysis, and pyrometers) on an optical table.

4. Align the red laser spot on the sample surface through the autoclave (and, if present, the glove box) window. If the laser spot is smaller than the sample surface, fix it in the center of the sample or in a particular area of interest (e.g., the interface between two different zones of the sample).

NOTE: The current high-power laser is also equipped with a low-power He-Ne red laser following exactly the same optical path. Turn on this pilot laser for aligning the system. The red laser spot size will be slightly different than the real spot size of the high-power infrared beam. However, the difference can be neglected in the alignment procedure.

5. Turn on the $\mathrm{Ar}^{+}$laser and align it in the center of the red pilot laser spot on the sample surface.

\section{Pyrometer alignment}

1. Fix the pyrometer and the spectro-pyrometer to the optical table in a position convenient for looking at the sample, with their axes as close as possible to perpendicular to the sample surface.

2. Roughly point the pyrometer and the spectro-pyrometer towards the sample. By looking through the respective eyepieces, make sure that the objectives see the sample correctly.

3. In order to finely align the pyrometer at the correct position and focal distance, shine a flexible lamp into the pyrometer eyepiece. Verify that a sharp image of the pyrometer diaphragm is projected onto the sample surface.

NOTE: In both the pyrometer and spectro-pyrometer, the thermal radiation emitted by the sample is collected by an objective (lenses and collimator) and focused on photodiode detectors through a diaphragm. The image of this diaphragm is clearly visible through the eyepieces of the pyrometers. With the current set-up, this spot is approximately circular, with a diameter of $1 \mathrm{~mm}$. Avoid playing with the pyrometer objective settings, because this might affect the device calibration.

4. Align the pyrometer diaphragm image in the middle of the red pilot laser spot and the $\mathrm{Ar}^{+}$blue laser spot.

5. Repeat the same procedure in steps 3.2.3-3.2.4 in order to finely align the spectro-pyrometer.

\section{Reflection check}

1. Carefully check for parasite reflections of the red pilot laser (apparent by eye with the help of a sheet of white paper), which mostly come from the autoclave (and, if present, the glove box) windows.

NOTE: These reflections might also originate from the sample surface, if a well-reflective metallic sample is to be analyzed. Such reflections are extremely dangerous when the sample is irradiated with the high-power infrared laser beam.

2. Place graphite screens (absorbers) wherever parasite reflections have been identified.

NOTE: IR laser beam reflections should never hit human beings, but they can also burn optical and electronic components and glove box parts, or they can be further reflected by metallic laboratory tools. Therefore, they should be stopped by adequate graphite absorbers as close as possible to their origin.

\section{Filling the pressure vessel}

1. Connect the pressure vessel to a vacuum pump and a gas supply system through suitable pipes. If possible, in addition to a manometer, connect an oxygen analyzer to the pressure vessel.

2. Choose the atmosphere (gas or gas mixture) under which laser-heating experiments should be performed. Select the atmosphere depending on the sample to be investigated and the chemical conditions to be produced.

3. Whatever the atmosphere, first use a vacuum pump to empty the pressure vessel, in order to avoid any cross-contamination with air, especially if the selected experimental atmosphere is ideally free of oxygen. If possible, attain the lower detection limit of the $\mathrm{O}_{2}$ analyzer in this "purging" procedure.

4. After emptying it, fill the autoclave with the chosen gas at the pressure required. 
NOTE: Different pressures can be set inside the autoclave (e.g., for studying the pressure effect on phase transitions). However, for standard experiments, set a gas overpressure of 0.2-0.3 $\mathrm{MPa}$ (with respect to atmospheric pressure) in order to reduce sample vaporization phenomena as much as possible. Most of the current experiments are performed under an inert atmosphere (pressurized argon) in order to maintain the initial sample composition throughout the laser-heating experiments. However, for special studies, oxidizing (pressurized air, CO/ $\mathrm{CO}_{2}$ mixtures, etc.) or reducing $\left(\mathrm{Ar}+\mathrm{H}_{2}\right)$ atmospheres can be employed, too.

5. After filling the autoclave, make sure that the oxygen potential has stabilized on the oxygen analyzer before starting the laser-heating experiment.

\section{Setting up the acquisition system}

1. Connect the two pyrometer channels ( $488 \mathrm{~nm}$ for the RLS and $650 \mathrm{~nm}$ for the temperature analyses) to an oscilloscope acting as an analogue/digital $(A D)$ converter.

2. Repeat step 5.1 for the spectro-pyrometer. NOTE: Due to its large number of channels, the spectro-pyrometer is equipped with its own acquisition unit. This can be triggered externally with a signal coming from the oscilloscope.

3. Connect the high-power laser potentiometer with the same oscilloscope as the pyrometers. Make sure that the oscilloscope has at least three input plugs. Otherwise, connect an additional device to it and synchronize them.

4. Set the oscilloscope parameters (acquisition window amplitude, offset, and sweeping duration) in such a way that the experimental data coming from the pyrometer can be correctly and entirely recorded. Check on the oscilloscope screen that data are correctly recorded and saved after each experiment.

5. Set a suitable trigger for the acquisition system. For example, trigger the oscilloscope when the signal coming from the laser potentiometer trespasses a certain threshold, corresponding to the onset of the first high-power pulse sent to the sample and set with the help of the oscilloscope software.

1. Verify that, as the oscilloscope is triggered, it starts to record the signals coming from the laser potentiometer and from the two pyrometer channels and also sends a signal that triggers acquisition in the spectro-pyrometer.

6. Connect the oscilloscope to a PC. Directly insert software calibration equations 2,3 and 4 so that the recorded intensity can be plotted directly as temperature-versus-time curves (thermograms) on the PC screen.

\section{Laser-heating shots}

1. Set a laser-heating program. If possible, do it directly from a PC connected to the laser.

2. For refractory materials melting beyond $2,500 \mathrm{~K}$, set up a pre-heating stage at the beginning of the laser program. This consists of a slowheating stage lasting 10 to $30 \mathrm{~s}$, during which the sample is heated with a low laser power density (around $50 \mathrm{~W} \mathrm{~cm}^{-2}$ ) until its temperature is stabilized at a constant level between 1,500 and $2,000 \mathrm{~K}$.

NOTE: The pre-heating stage reduces thermal stresses, which could easily crack and destroy the sample if it was fired directly to over 2,500 $\mathrm{K}$ starting from room temperature. In addition, it helps remove possible impurities from the sample surface. For laser melting experiments, the best approach has been established based on direct experience.

3. After the pre-heating stage, set up a sequence of several higher-power laser shots, heating the sample well beyond its melting temperature. Define cycles of 3-4 shots, after which the sample can cool back to room temperature. Check the sample conditions before proceeding to further shots.

NOTE: The sample should not be allowed to cool back to room temperature in between two shots in order to avoid too-intense thermal stresses. The power required varies depending on the laser spot and the investigated material. Typically, for refractory oxides like $\mathrm{UO}_{2}$, power densities of approximately $500 \mathrm{~W} \mathrm{~cm}^{-2}$ are sufficient to melt the material surface in a few hundred ms.

4. Vary the duration of successive high-power laser pulses (and the respective power density) between a few tens of ms and a few $\mathrm{s}$ in order to check for the possible dependence of the observed thermal arrest temperatures on the pulse length. In this way, verify whether phase transitions occur at the thermodynamic equilibrium during the heating/cooling cycles.

NOTE: No thermodynamic equilibrium conditions would be ensured with shorter pulses, whereas longer pulses should be avoided because the liquid mass would no longer be kept on the sample surface by capillarity forces, and by falling, it would damage the sample containment (holder and autoclave).

5. During the laser-heating experiments, stay in a control room separated from the main laboratory by coated protective windows that stop the high-power laser radiation.

NOTE: If experimenter presence is needed in the main laboratory during the laser shots, it is imperative to wear protective glasses.

6. Verify that the set-up laser program works properly by first shooting the laser beam in a graphite absorber. Use this test to also check that the function of the trigger system arranged in step 5.5 is correct.

7. If all checks are successful, deactivate the red pilot laser and switch on the high-power beam.

8. Release all safety switches and start the laser irradiation program on the sample.

9. At the end of the laser-heating and cooling cycles (typically the pre-heating stage plus three or four high-power pulses), verify the appearance of the sample, indicating whether it is totally or partially melted, quenched, broken, still intact, etc.

10. If the sample is still intact, repeat several laser-heating cycles on it and check for result repeatability. NOTE: In successful cases, over forty shots can be repeated on the same sample. Such large datasets can be treated, yielding average values for the phase transition points supported by a sound statistical analysis of the measurement uncertainty.

\section{Data analysis}

\section{Qualitative thermogram analysis}


1. Check the quality and features of experimental thermograms (one per laser shot) recorded by the pyrometer. Verify that, if the maximum temperatures reached were high enough, thermal arrests corresponding to solidification appear on the cooling parts of the thermograms.

NOTE: Similar thermal arrests are usually hardly visible on the heating flank because the fast laser heating provides more energy than the melting enthalpy and thermodynamic equilibrium conditions are mostly not realized in this part of the experiment ${ }^{11}$.

2. If the maximum temperatures were too low, repeat the laser-heating cycle with higher-power pulses.

3. Repeat the laser-heating/cooling cycles on a new sample if thermograms are too irregular or aberrant (e.g., the apparent heating and cooling of the sample do not follow the laser pulses), in which case, the sample probably broke, cracked, or vaporized during the experiment.

\section{Emissivity analysis}

1. In order to obtain real sample temperature thermograms, establish, with the help of the spectro-pyrometer data, the NSE of the sample.

2. Transform raw spectro-pyrometer data into radiance spectra, as explained in step 1.4.11.

3. If a well-established temperature point $T^{\star}$ exists (e.g., a reference eutectic point [Ref. ZrC-C]) in the investigated system and is measured in the current experiment, then obtain NSE directly from real body radiance spectra $L_{\lambda, r b}$ measured on the corresponding thermal arrest. Knowing the real temperature $T^{\star}$ at which the thermal arrest occurs, calculate radiance $L_{\lambda, r b}$ through Equation 1 . In this case, obtain the emissivity directly from its definition as:

$$
\varepsilon_{\lambda}(\lambda, T)=\frac{L_{\lambda, r b}\left(\lambda, T^{*}\right)}{L_{\lambda, b b}\left(\lambda, T^{*}\right)} \quad \text { Equation } 9 .
$$

4. If no established temperature points are available, then fit radiance spectra $L_{\lambda, r b}$, taking NSE $\varepsilon_{\lambda}$ and temperature $T$ as free parameters in Equation 1. Then, obtain emissivity and temperature as the best values that fit the whole spectrum.

NOTE: This procedure is numerically accurate if the grey body assumption is valid (i.e., if emissivity does not depend on wavelength, which is mostly true for the materials investigated in this work). Otherwise, further assumptions on the emissivity wavelength dependence are needed in order to estimate a parametric dependence of $\varepsilon_{\lambda}$ on $\lambda$ with the help of literature data.

5. Once $\varepsilon_{\lambda}$ is determined, multiply its value at $650 \mathrm{~nm}$ by the optical transmittance of the pressure vessel (and, if present, the glovebox) window at $650 \mathrm{~nm}$ (provided by the window supplier) and substitute it in Equation 3. In this way, obtain real temperature thermograms from the pyrometer measurements.

\section{Phase transition study}

1. Identify phase transition points as the temperatures at which thermal arrests or inflections occur in the cooling flank of real temperature thermograms.

2. Perform the first laser-heating experiments on refractory materials, the melting points and NSE values of which are well established (e.g., Molybdenum, tungsten, $\mathrm{ZrC}$, or $\mathrm{UO}_{2}$ ). This will provide a sound test of the good functioning and accuracy of the method. NOTE: Leave out from the analysis possible inflections, if any, on the heating flank of the thermograms, as they may be the result of non-equilibrium phenomena of very uncertain interpretation.

3. Compare the RLS signal, simultaneously displayed, with the real temperature thermograms. Identify the onset of new phases on the sample surface with the help of the RLS, where oscillations and inflections will appear correspondingly.

4. Compare phase transition temperatures recorded by the pyrometer with eventual NSE changes related to the same phase transitions.

\section{Sample recovery}

1. Release the pressure from the autoclave and stabilize it to atmospheric pressure.

2. Open the autoclave and remove the melted and refrozen sample, as well as possible fragments that have fallen off. Possibly use these parts for post-melting material characterization.

3. Clean the autoclave carefully, especially the optical windows, with the help of tissues and ethanol.

4. Collect samples and fragments in adequate containers. In case of glove box work with highly-radioactive samples, place the containers inside a lead box.

\section{Representative Results}

Figure 3 displays real temperature thermograms measured on uranium dioxide with various oxidation levels $\left(\mathrm{UO}_{2+x} \text { with } 0<x<0.21\right)^{2}$. Uranium dioxide is the essential component of the most common fuel in current NPPs. Its oxidation to various oxygen hyper-stoichiometry levels can occur in normal and off-normal reactor conditions ${ }^{12}$. With the current method, it was shown that $\mathrm{UO}_{2}$ oxidation can result in a dramatic decrease of its melting/solidification point by up to $700 \mathrm{~K}$. In this case, experiments had to be carried out under a rather high inert gas pressure (He at 10 $\mathrm{MPa}$ ) in order to suppress the highly non-congruent vaporization at high temperatures. 


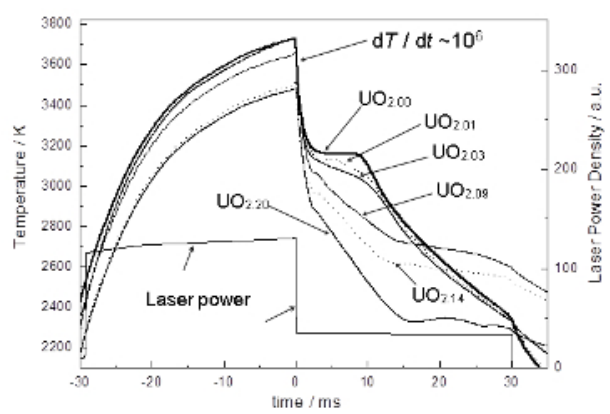

Figure 3: Thermograms measured on laser-heated stoichiometric and hyperstoichiometric uranium dioxide samples (after ${ }^{2}$ ).

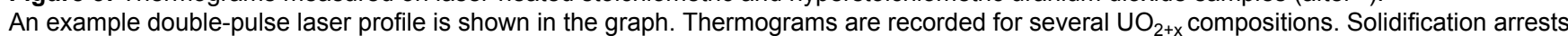
occur at significantly-different temperatures and with different features, depending on the sample composition, revealing the evolution of the melting/freezing temperature and solidification dynamics in the U-O system. Please click here to view a larger version of this figure.

Figure 4 shows pyrometer (straight line) and spectro-pyrometer thermograms recorded on a plutonium dioxide sample laser heated under an oxidizing atmosphere (compressed air at $0.3 \mathrm{MPa}$ ). Also, $\mathrm{PuO}_{2}$ is an essential nuclear fuel component. In the same figure, two radiance spectra measured by the spectro-pyrometer at different temperatures are also displayed in the insets, together with curves fitting experimental data and the corresponding $T$ and $\varepsilon_{\lambda}$ values. Thanks to the present study, the $\mathrm{PuO}_{2}$ melting/freezing temperature was reassessed to be $3,017 \mathrm{~K} \pm 28 \mathrm{~K}$, over $300 \mathrm{~K}$ higher than previously indicated by more traditional heating methods. Those methods yielded results certainly affected by extensive high-temperature interactions between the sample and containment, an issue that has been largely solved with the present remote heating approach.

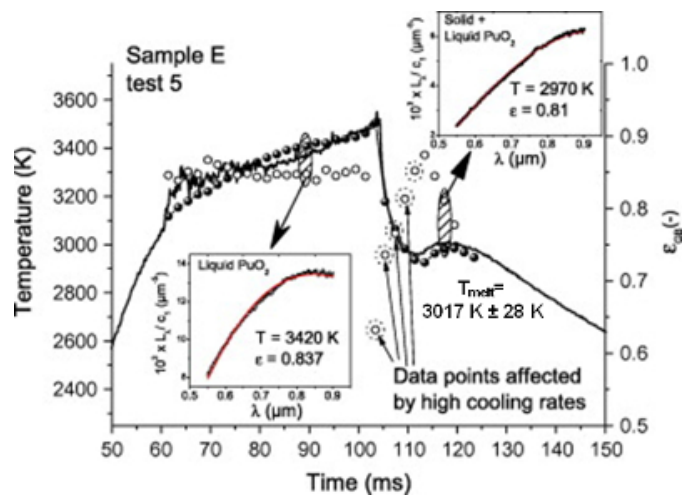

Figure 4: Thermograms measured on a plutonium dioxide sample laser heated beyond the melting point.

Main graph: the black solid line and the full black circles represent the thermograms recorded on a $\mathrm{PuO}_{2}$ sample under an oxidizing atmosphere by the fast pyrometer and the multi-wavelength spectro-pyrometer, respectively. The white circles represent the spectral emittance values obtained by fitting experimental radiance data with Planck's radiance law ${ }^{12}$. The two insets show example spectra recorded (black circles) and fitted (red solid lines) in liquid and freezing $\mathrm{PuO}_{2}$, respectively, within the grey body assumption. In these plots, the radiance $L_{\lambda}$ is normalized to the first radiation constant $c_{1}$ for the sake of simplicity. The main thermogram was obtained using an average constant emittance of 0.83 . Please click here to view a larger version of this figure.

Figure 5 shows a series of laser-heating pulses performed on a mixture of $\mathrm{UO}_{2}$ and $\mathrm{ZrO}_{2}$ under different atmospheres. This test is representative of conditions that may be produced in case of an accidental temperature excursion in a NPP. The melting/solidification point occurs at a wellrepeatable temperature over successive shots if experiments are carried out in argon. On the other hand, the melting/solidification temperature decrease over the laser shots if laser-heating cycles are performed in compressed air. This shows that, in the latter case, the sample gets increasingly oxidized during the laser-heating treatments. Also, in the case of mixed $\mathrm{UO}_{2}-\mathrm{ZrO}_{2}$ oxides, a melting point depression occurs in oxygen hyper-stoichiometry conditions. 


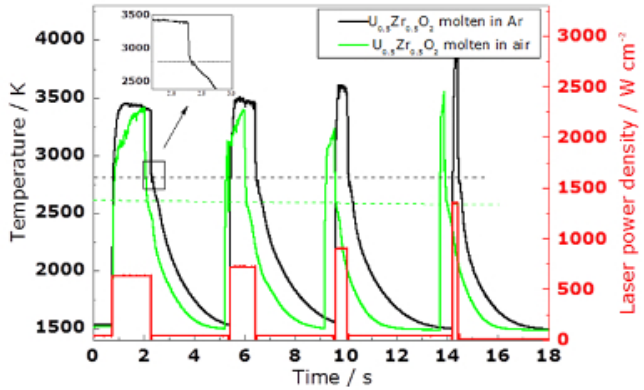

Figure 5: Thermograms measured mixed $\mathrm{UO}_{2}-\mathrm{ZrO}_{2}$ samples in pressurized argon and air.

The melting/solidification point occurs at a well-repeatable temperature over successive shots if experiments are carried out in argon (black thermograms). On the other hand, the melting/solidification temperature decrease over the laser shots if laser-heating cycles are performed in compressed air (green thermograms). Please click here to view a larger version of this figure.

A further example concerns another kind of material, uranium dicarbide. This is envisaged as a possible material for an alternative concept of nuclear fuel, potentially working at higher temperatures and considerably reducing the risk of a meltdown accident. A new composition containing a large excess of carbon (nominally $\mathrm{UC}_{2.8}$ ) was investigated for the first time with the current approach ${ }^{14}$. In this case, the $\mathrm{UC}_{2}$ - $\mathrm{C}$ eutectic temperature, established to be at $27,37 \mathrm{~K} \pm 20 \mathrm{~K}$, was used as a radiance reference together with the cubic-tetragonal ( $\alpha \rightarrow \beta$ ) solid-state transition, fixed at $2,050 \mathrm{~K} \pm 20 \mathrm{~K}$. The NSE of the carbon-richer compound was measured to increase up to 0.7 at $650 \mathrm{~nm}$, whereas the value $\varepsilon_{\lambda}$ $=0.53$ was established for pure uranium dicarbide at the limit of the eutectic region. This increase was analyzed in light of the demixing of excess carbon and used for the determination of the liquidus temperature $\left(3,220 \pm 50 \mathrm{~K}\right.$ for $\left.\mathrm{UC}_{2.8}\right)$. Due to fast solid-state diffusion, also fostered by the cubic-tetragonal transition, no obvious signs of a lamellar eutectic structure could be observed after quenching to room temperature. The eutectic surface $\mathrm{C} / \mathrm{UC}_{2-\mathrm{x}}$ composition could be qualitatively, but consistently, followed during the cooling process with the help of the recorded radiance spectra, as shown in Figures 6 a and b. Interestingly, the current NSE analysis showed that, whereas in the liquid phase the external liquid surface was almost entirely constituted of uranium dicarbide, it got rapidly enriched in demixed carbon upon freezing. Demixed carbon seemed to quickly migrate towards the inner bulk during further cooling. At the $\alpha \rightarrow \beta$ transition, uranium dicarbide again covers almost the entire external surface. All of these details on the very high-temperature material behaviour are essential for the analysis of this type of compound in case of uncontrolled temperature increase in the reactor core. They were deduced only on the basis of radiance spectroscopy analysis, whereas they would be hardly accessible to any other experimental investigation technique.
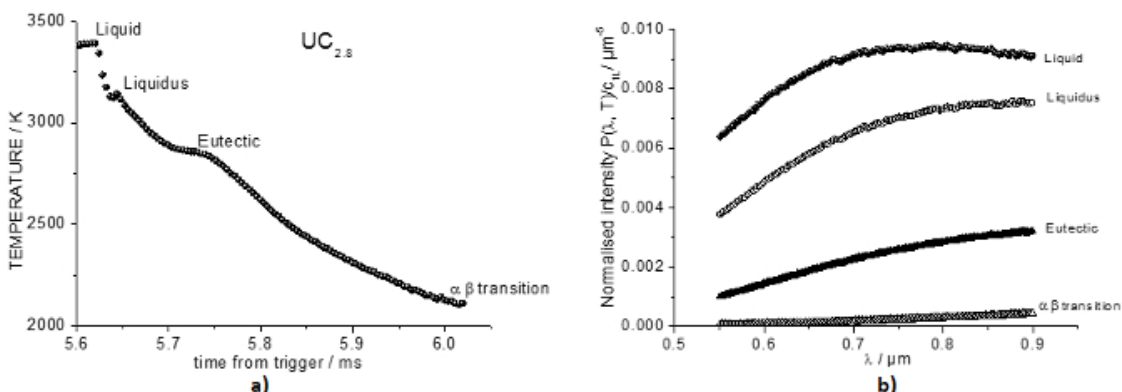

b)

Figure 6: Thermogram and radiance spectra measured on a $\mathrm{UC}_{2.8}$ sample in pressurized argon ${ }^{14}$.

a) The cooling stage of a thermogram recorded on a $\mathrm{UC}_{2.8}$ sample. Full circles identify the time points at which the radiance spectra were recorded by the spectro-pyrometer. b) Four examples of radiance spectra recorded at different temperatures. One of them was recorded in liquid $\mathrm{UC}_{2.8}$, while the other three correspond to the thermal arrests visible in Figure 5a. Please click here to view a larger version of this figure.

\section{Discussion}

The laser-heating radiation spectroscopy technique presented here is recognized as an innovative and effective method for the investigation of very high-temperature and melting behavior of refractory materials ${ }^{15,16}$. Thanks to its remote and almost container-less nature, it is particularly suited for the experimental study of radioactive nuclear materials and the simulation of core meltdown accidents in NPPs, as shown by the example results presented here.

While evaluating experimental data obtained with the current method, one should no doubt be careful about the correct assignment of experimental points to phase transitions. In fact, at very high temperatures, material kinetics can be extremely fast, and several difficult-tocontrol phenomena may occur, such as non-congruent vaporization, segregation, compound dissociation, etc. As the comparison with more traditional heating methods (like induction furnaces) demonstrates, the possible occurrence of such phenomena justifies the use of a fast heating and cooling technique like the current one. On the other hand, doubts may arise about the effective stabilization of thermodynamic equilibrium conditions under the current heating conditions. As explained in the procedure section, such conditions cannot be guaranteed during the fast laser-heating part of the thermal cycles. However, thermodynamic equilibrium conditions are certainly produced on the cooling stage. This statement was validated with the help of computer codes simulating the current experiments and based on near-equilibrium mass and heat diffusion in the presence of local phase transitions ${ }^{11}$. Nonetheless, thermodynamic equilibrium conditions should always be cross- 
checked experimentally, typically by measuring well-assessed phase transition temperatures in compounds that can be taken as references. This was realized in the present work with the melting/solidification points of W, Mo (recommended as secondary reference temperatures in the International Temperature scale of $\left.1990^{17,18,19}\right), \mathrm{UO}_{2}$, and the $\mathrm{ZrC}-\mathrm{C}$ eutectic ${ }^{9}$. Measuring such reference points is also necessary in order to assess the accuracy and uncertainty of the present approach.

Given the extreme conditions and phenomena produced in the laser-heating experiments, a precise uncertainty analysis is paramount for the usability of the data produced. For successful measurement campaigns, the cumulative uncertainty affecting the current phase transition temperature data should amount to $\pm 1 \%$ of the absolute temperature, with a 2 -standard-deviation coverage factor (95\% confidence). Such uncertainty bands can be larger for complicated materials, where, for example, non-congruent vaporization may change the actual sample composition in an uncontrollable way during the experiments. Such uncertainty should take into account the errors due to the calibration procedure, the NSE determination, the sample stability (i.e., the repeatability, over successive laser shots, of experimental phase transition temperatures), etc. An example of uncertainty analysis for the melting/freezing point of $\mathrm{PuO}_{2}$ is reported in Table 1. The various uncertainty contributions can be considered as independent and combined according to the error propagation law ${ }^{3}$.

\begin{tabular}{|c|c|c|c|}
\hline $\begin{array}{l}\qquad \delta T_{\varepsilon} \\
\text { due to experimental standard deviation on } \\
\text { emissivity } \Delta \varepsilon_{\lambda}=0.04 \text {, with average } \varepsilon_{\lambda}=0.825 \\
\text { and average } T_{m}=3017 \mathrm{~K} \text {. } \\
\delta T_{\epsilon}=T_{m i}^{2} \cdot \frac{\lambda}{c_{2}} \cdot \frac{\Delta s_{\lambda}}{\varepsilon_{\lambda}}\end{array}$ & $\begin{array}{l}\qquad \delta T_{\epsilon} \\
\text { due to intrinsic calibration } \\
\text { uncertainty (reference light sources) } \\
\text { extrapolated at } 3000 \mathrm{~K}\end{array}$ & $\begin{array}{l}\delta T_{\rho} \\
\text { due to experimental } \\
\text { data dispersion }\end{array}$ & $\delta T_{\text {tox }}=\sqrt{\delta T_{f}^{2}+\delta T_{c}^{2}+\delta T_{d}^{2}}$ \\
\hline $\pm 20.09 \mathrm{~K}$ & $\pm 10 \mathrm{~K}$ & $\pm 17 \mathrm{~K}$ & $\pm 27.87 \mathrm{~K}$ \\
\hline
\end{tabular}

Table 1: Example of uncertainty analysis for the melting/freezing point of $\mathrm{PuO}_{2}$ (Reference ${ }^{13)}$.

The meaning and value of $\mathrm{c}_{2}$ is reported in the introduction section with the comments on Equation 1. $\Delta \varepsilon_{\lambda}$ stands here for two standard deviations around the average experimental value obtained for $\varepsilon_{\lambda}$ by fitting experimental radiance spectra within the grey body assumption. $\delta T_{c}$ and $\delta T_{d}$ represent two standard deviations around the average standard lamp extrapolated temperature curve and the average experimental solidification temperature value, respectively.

Some improvements can be made on the present experimental approach. In particular, connecting the pressure vessel with a mass spectrometer through a complex pipe system will allow the detection, at least qualitatively, of the species present in the vapor plume released by the hot material. Moreover, the implementation of a thermo-camera is foreseen for the two-dimensional study of the temperature distribution over the hot sample surface in order to detect possible inhomogeneities and segregation effects. Finally, improvements in the safety system built around the current equipment are foreseen. Actually, the current Plexiglas glove box used here is suited for the study of highly-radioactive materials, such as uranium and transuranium elements, thanks to the fact that it effectively blocks a radiation. However, this shield is not sufficiently safe for the investigation of strong y emitters, like the nuclides contained in real irradiated nuclear fuel. A new facility including a lead-walled cell is foreseen for the study of spent nuclear fuel coming from real NPPs.

\section{Disclosures}

The authors have nothing to disclose.

\section{Acknowledgements}

The authors are indebted to the European Commission for funding the present research under its institutional research programs. In addition, part of the presented research was financed through the EC $6^{\text {th }}$ Framework Program under the F-BRIDGE project and $7^{\text {th }}$ FP under the SAFEST and GENTLE projects.

\section{References}

1. Council Directive 2009/71/Euratom of 25 June 2009 establishing a Community framework for the nuclear safety of nuclear installations. http:/l eur-lex.europa.eu/legal-content/EN/TXT/?qid=1412848109512\&uri=CELEX\%3A32009L0071 (2009).

2. Manara, D., Ronchi, C., Sheindlin, M., Lewis, M., Brykin, M., Melting of stoichiometric and hyperstoichiometric uranium dioxide, J. Nucl. Mater. 342 148-163 (2005).

3. Manara, D., Sheindlin M., Heinz W., Ronchi C., New techniques for high-temperature melting measurements in volatile refractory materials via laser surface heating, Review of Scientific Instruments. 79 113901-08 (2008).

4. De Bruycker, F., et al. Reassessing the melting temperature of PuO2, Materials Today. 13, $52-55$ (2010).

5. Kato, M., et al. Solidus and liquidus temperatures in the $\mathrm{UO}_{2}-\mathrm{PuO}_{2}$ system, J. Nucl. Mater. 373 237-245 (2008).

6. DeWitt, D.P., Richmond, J.C. Thermal radiative properties of materials. In: DeWitt, D.P., Nutter, G.D., editors. Theory and practice of radiation thermometry. New York: Wiley; (1988).

7. Neuer, G., Fiessler, L., Groll, M., Schreiber, E., Critical analysis of the different methods of multiwavelength pyrometry. In: Schooley JF, editor. Temperature: its measurement and control in science and industry, vol. 6. New York: AIP, 787-789 (1992).

8. Jacquemain, D., et al. (co-ordinator), Nuclear Power Reactor Core Melt Accidents. State of Knowledge. EDP Science. ISBN: 978-2-7598-1835-8 (2015).

9. Manara, D., et al., The ZrC-C eutectic structure and melting behaviour: A high-temperature radiance spectroscopy study, J. Eur. Ceram. Soc. 33, 1349-1361 (2013).

10. Manara, D., et al., On the melting behaviour of calcium monoxide under different atmospheres: A laser heating study, J. Eur. Ceram. Soc. $\mathbf{3 4}$ 1623-1636 (2014). 
11. Welland, M.J., Thompson, W.T., Lewis, B.J., Manara, D., Computer simulations of non-congruent melting of hyperstoichiometric uranium dioxide, J. Nucl. Mater. 385, 358-363 (2009).

12. Olander, D., Nuclear Fuels- Present and future, J. Nucl. Mater., 389, 1-22 (2009).

13. De Bruycker, F., et al. The melting behaviour of plutonium dioxide: A laser-heating study. J. Nucl. Mater., 416, 166-172 (2011).

14. Manara, D., Boboridis, K., Morel, S., De Bruycker, F., The $\mathrm{UC}_{2-\mathrm{x}}$ - Carbon eutectic: A laser heating study, J. Nucl. Mater., 466, 393-401 (2015).

15. Barrachin, M., Chevalier, P.Y., Cheynet,B., Fischer,E., New modelling of the U-O-Zr phase diagram in the hyper-stoichiometric region and consequences for the fuel rod liquefaction in oxidising conditions, J. Nucl. Mater., 375, 397-409 (2008).

16. Guéneau, C., Chartier, A., Van Brutzel, L., Thermodynamic and thermophysical properties of the actinide oxides, Comp Nucl Mater. 2, 21-59 (2012).

17. Preston-Thomas, H., The International Temperature Scale of 1990 (ITS-90), Metrologia. 27, 3-10 (1990).

18. Preston-Thomas, H. Erratum: The International Temperature Scale of 1990 (ITS-90). Metrologia. 27, 107 (1990).

19. Bedford, R. E., Bonnier, G., Maas, H., Pavese, F. Recommended values of temperature on the International Temperature Scale of 1990 for a selected set of secondary reference points, Metrologia. 33,133-154 (1996). 